POS PROCEEDINGS

\title{
Role of Light-Front Coordinates in String Theory
}

\author{
Daya Shankar Kulshreshtha*† \\ Department of Physics and Astrophysics, \\ University of Delhi, Delhi-110007, India. \\ E-mail: dskulsh@gmail.com

\section{Usha Kulshreshtha} \\ Department of Physics, Kirori Mal College \\ University of Delhi, Delhi-110007, India. \\ E-mail: ushakulshegmail.com
}

\begin{abstract}
In this work, we present a review of the role of light-front coordinates (LFC's) in string theory and also a review of the role of light-front (LF) zero modes (ZM's) in string theory as covered in my two talks at LC2019. It is seen that the light-front coordinates play a central role in the understanding of not only the bosonic string theory (ST) but also in the understanding of the superstring theory (SST) that includes fermionic string fields. First we consider the bosonic string sigma model action known as the Polyakov action and study it in the so-called conformal gauge using the LFC's. The equation of motion (EoM) and the components of the conserved energy momentum tensor are solved in the LFC's. We introduce the LF Fourier modes for the left movers and right movers. The LF Fourier modes for the energy momentum tensor are the Virasoro operators. The Virasoro operators corresponding to the LF zero modes are seen to play a central role in obtaining the mass spectrum of ST and in determining the number of spacetime dimensions of the critical ST. We try to illustrate these important concepts using the LFC's. This eventually also helps in understanding the concept of the compactification of extra dimensions in ST. We then consider the Raymond-Nevieu-Schwarz (RNS) SST in LFC's and study its conserved super current, conserved energy-momentum tensor, EoM and the Raymond and Nevieu-Schwarz boundary conditions that are needed to make the boundary terms vanish. We then discuss that the consistency of the SST in the R-sector as well as separately in the NS-sector requires the number of spacetime dimensions of the SST to be $D=10$ it also leads to the results that the spectrum of the SST in the R-sector leads to spacetime fermions and the spectrum of the theory in the NS-sector leads to spacetime bosons in $D=10$. dimensions.
\end{abstract}

Light Cone 2019 - LC2019

September 16-20, 2019

Ecole Polytechnique Palaiseau, France

\footnotetext{
${ }^{*}$ Speaker.

${ }^{\dagger}$ Invited Talk.
} 
In this work, we present a review of the role of light-front (LF) coordinates (LFC's) [1, 2] in bosonic string theory (ST) and superstring theory (SST) [3]-[8] and also a review of the role of LF zero modes (ZM's) in ST [3]-[8], as covered in my two talks. It is seen that the LFC's play a central role in the understanding of not only the bosonic ST [8] but also in the understanding of the SST [8]. First we consider the bosonic string sigma model action also known as Polyakov action in conformal gauge and study the mass spectrum of bosonic ST using LF Fourier modes of left and right movers [3]-[8]. We also present a review of the basics of RNS SST in LFC's [3]-[8]. The string sigma model action popularly known as the Polyakov action is defined by [3]-[8]:

$$
\tilde{S}_{B}=\int \tilde{\mathscr{L}}_{B} d^{2} \sigma, \tilde{\mathscr{L}}_{B}=\left[-\frac{T}{2} \sqrt{-h} h^{\alpha \beta} G_{\alpha \beta}\right], G_{\alpha \beta}=\partial_{\alpha} X^{\mu} \partial_{\beta} X^{v} \eta_{\mu v}, h=\operatorname{det}\left(h_{\alpha \beta}\right)
$$

Here, $\eta_{\mu v}=\operatorname{diag}(-1,+1, \ldots,+1) \quad, \quad \mu, v=0,1, \ldots,(d-1) ; \alpha, \beta=0,1$. Also, $\sigma^{\alpha} \equiv(\tau, \sigma)$ are the two parameters describing the world-sheet (WS). The overdots and primes would denote the derivatives with respect to $\tau$ and $\sigma . T$ is the string tension. $G_{\alpha \beta}$ is the induced metric on the WS and $X^{\mu}(\tau, \sigma)$ are the maps of the WS into the $d$-dimensional Minkowski space and describe the strings evolution in spacetime and $h_{\alpha \beta}$ are the 2 dimensional (2D) auxiliary fields. The theory could be studied using the Dirac's instant-form (IF) of dynamics on the hyperplanes defined by the WS time $\sigma^{0}=\tau=$ constant $[1,2]$ or by using the front-form (FF) or the light-front (LF) dynamics on the hyperplanes of the LF defined by the LF-WS time $\sigma^{+}=(\tau+\sigma)=$ constant [1,2]. Variation of the above action with respect to the auxiliary field $h_{\alpha \beta}$ implies that the energy-momentum tensor:

$$
T_{\alpha \beta}=\left[G_{\alpha \beta}-\frac{1}{2} h_{\alpha \beta}(h \cdot G)\right]=0, \quad h . G=\left(h^{\gamma \delta} G_{\gamma \delta}\right) .
$$

Also, we define the LFC's as [1,2]:

$$
\sigma^{ \pm}:=(\tau \pm \sigma), \partial_{ \pm}:=\frac{1}{2}\left(\partial_{\tau} \pm \partial_{\sigma}\right), \partial^{ \pm}=\left(-2 \partial_{\mp}\right), X^{ \pm}=\left(X^{0} \pm X^{1}\right) / \sqrt{2} .
$$

The theory is seen to possess three local gauge symmetries due to WS reparametrization invariance (WSRI) and the Weyl invariance (WI) the details of which are omitted here for the sake of brevity $[1,2,3]$. In the conformal gauge (CG), the auxiliary field $h_{\alpha \beta}$ turns out to be proportional to the metric tensor $\eta_{\alpha \beta}$ of the WS and the proportionality constant could be taken equal to one in CG [3]-[8]. The three local gauge symmetries of the theory given by the WSRI and WI could be used to fix the components of $h_{\alpha \beta}$ (because one could use these local gauge symmetries of the theory to choose $h_{\alpha \beta}$ to be of a particular form: $h_{\alpha \beta}:=\eta_{\alpha \beta}, h^{\alpha \beta}:=\eta^{\alpha \beta}$. In the instant-form (IF) dynamics we take $[1,2]$ :

$$
h_{\alpha \beta}=\eta_{\alpha \beta}=\left(\begin{array}{cc}
-1 & 0 \\
0 & +1
\end{array}\right), h^{\alpha \beta}=\eta^{\alpha \beta}=\left(\begin{array}{cc}
-1 & 0 \\
0 & +1
\end{array}\right), \sqrt{-h}=\sqrt{-\operatorname{det}\left(h_{\alpha \beta}\right)}=1
$$

and in the LF dynamics we take [3]-[8]:

$$
h_{\alpha \beta}:=\eta_{\alpha \beta}=\left(\begin{array}{cc}
0 & -1 / 2 \\
-1 / 2 & 0
\end{array}\right), h^{\alpha \beta}:=\eta^{\alpha \beta}=\left(\begin{array}{cc}
0 & -2 \\
-2 & 0
\end{array}\right), \sqrt{-h}=\sqrt{-\operatorname{det}\left(h_{\alpha \beta}\right)}=1 / 2 .
$$

The action of the theory in the CG reads:

$$
S_{B}=\int \mathscr{L}_{B} d^{2} \sigma, \mathscr{L}_{B}=\left[(-T / 2)\left(\partial_{\alpha} X^{\mu} \partial^{\alpha} X_{\mu}\right)\right] .
$$


Here, $\alpha=0,1(I F), \mu=0,1,2,3, \ldots, 25(I F)$ and $\alpha=+,-(L F), \mu=+,-, 2,3, \ldots, 25(L F)$. Variational principle applied to the above action implies an equation of motion (EoM): $\square X^{\mu}=0$ and some boundary terms which have to be made to vanish by the suitable choice of boundary conditions (BC's). The closed strings (CS) have periodic BC's whereas the open strings (OS) have two possibilities: (i) called the Neumann BC's (NBC's) for which we set the space derivatives of $X^{\mu}$ to be equal to 0 (i.e. $\partial_{\sigma} X^{\mu}=0$ ) (here the string can oscillate and its end points can move along the boundaries as long as their space derivatives vanish at the boundaries) and (ii) called the Dirichlet BC's (DBC's) for which we set the string coordinates $X^{\mu}=$ Constant (or $\delta X^{\mu}=0$ ) (here the string can oscillate but its end points remain fixed at the boundary).

The EoM: $\square X^{\mu}=0$ in LFC's imples: $\partial_{+} \partial_{-} X^{\mu}=0$. The non-vanishing components of the energy momentum tensor in LFC's read: $T_{++}=\left(\partial_{+} X^{\mu} \partial_{+} X^{\mu}\right)$ and $T_{--}=\left(\partial_{-} X^{\mu} \partial_{-} X^{\mu}\right)$. The general solution of the EoM could be expressed as: $X^{\mu}=\left[X_{L}^{\mu}\left(\sigma^{+}\right)+X_{R}^{\mu}\left(\sigma^{-}\right)\right]$where the left mover (LM): $X_{L}^{\mu}\left(\sigma^{+}\right)$depends only on ' $\sigma^{+}$and the right mover (RM): $X_{R}^{\mu}\left(\sigma^{-}\right)$depends only on $\sigma^{-}$. In fact, $X_{L}^{\mu}$ and $X_{R}^{\mu}$ could be thought of in the $(\tau, \sigma)$ coordinate system, as the left moving and right moving waves respectively. We could now move from classical ST to quantum ST using the following Fourier mode expansions (with $\alpha^{\prime}=l_{s}^{2} / 2=1 /(2 \pi T)$ ) :

$$
\begin{aligned}
& X_{L}^{\mu}\left(\sigma^{+}\right)=\frac{1}{2} x_{L}^{\mu}+\frac{1}{2} l_{s}^{2} \sigma^{+} p_{L}^{\mu}+\frac{i l_{s}}{\sqrt{2}} \sum_{k \neq 0} \frac{1}{k} \alpha_{k}^{\mu} e^{\left(-i k \sigma^{+}\right)} . \\
& X_{R}^{\mu}\left(\sigma^{-}\right)=\frac{1}{2} x_{R}^{\mu}+\frac{1}{2} l_{s}^{2} \sigma^{-} p_{R}^{\mu}+\frac{i l_{s}}{\sqrt{2}} \sum_{k \neq 0} \frac{1}{k} \beta_{k}^{\mu} e^{\left(-i k \sigma^{-}\right)} .
\end{aligned}
$$

Here $k$ is the wave number which for the CS theory as well as for both the cases of OS theory eventually turns out to be an integer say $n$ (so that $k=n=$ integer) which can take all values from $-\infty$ to $+\infty$ including zero. The terms corresponding to $n=0$ correspond to the so-called zero modes (ZM's) and these terms could be separated out from the summation terms in the above mode expansions. We could write:

$$
X^{\mu}=\left(X_{L}^{\mu}+X_{R}^{\mu}\right)=\left(x^{\mu}+l_{s}^{2} \tau p^{\mu}\right)+\frac{i l_{s}}{\sqrt{2}} \sum_{n \neq 0} \frac{1}{n}\left(\alpha_{n}^{\mu} e^{-i n \sigma}+\beta_{n}^{\mu} e^{-i n \sigma}\right) e^{-i n \tau}
$$

Here $x^{\mu}$ is the center of mass (c.o.m.) position of the string and $p^{\mu}$ is the c.o.m. total momentum of the string. The first part in the above expansion describes the c.o.m. motion of the string (which does not depend on $\sigma$ ) and the string moves around in spacetime with this part and the string oscillates in spacetime with the second part which is periodic in $\sigma$. For quantum ST, we promote all the variables in the above equations to quantum mechanical operators as per rules of quantum mechanics (QM). The reality condition on $X^{\mu}$ implies: $X^{\mu}=\left(X^{\mu}\right)^{\star}=\left(X^{\mu}\right)^{\dagger}$ which in turn implies: $\quad x^{\mu}=\left(x^{\mu}\right)^{\star}, \quad p_{L}^{\mu}=\left(p_{L}^{\mu}\right)^{\star}, \quad p_{R}^{\mu}=\left(p_{R}^{\mu}\right)^{\star}$. Creation operators are: $\left(\alpha_{n}^{\mu}\right)^{\dagger}=\left(\alpha_{-n}^{\mu}\right)$ and $\left(\beta_{n}^{\mu}\right)^{\dagger}=\left(\beta_{-n}^{\mu}\right)$ and the annihilation operators are: $\alpha_{n}^{\mu}$ and $\beta_{n}^{\mu}$. ZM's of the theory correspond to $n=0$ and for these ZM's we have: $\alpha_{0}^{\mu}:=\frac{l_{s}}{\sqrt{2}} p_{L}^{\mu}=\sqrt{\alpha^{\prime}} p_{L}^{\mu}$ and $\beta_{0}^{\mu}:=\frac{l_{s}}{\sqrt{2}} p_{R}^{\mu}=\sqrt{\beta^{\prime}} p_{R}^{\mu}$.

Higher energy states could be built with the application of the creation operators on the ground state of the system. The non-vanishing canonical commutation relations of the theory are:

$$
\left[\alpha_{m}^{\mu}, \alpha_{n}^{v}\right]=\left[\beta_{m}^{\mu}, \beta_{n}^{v}\right]=m \eta^{\mu v} \delta_{m+n, 0}, \quad\left[x^{\mu}, p^{v}\right]=\eta^{\mu v}
$$


This in particular, implies: $\left[\alpha_{m}^{0}, \alpha_{-m}^{0}\right]=\left[\beta_{m}^{0}, \beta_{-m}^{0}\right]=m \eta^{00}=-m$. The theory is therefore seen to have the negative-norm states in its spectrum. Number operators for the left and right movers are: $N_{L}:=\left(\alpha_{n}^{\mu}\right)^{\dagger}\left(\alpha_{n}^{\mu}\right)=\left(\alpha_{-n}^{\mu}\right)\left(\alpha_{n}^{\mu}\right), N_{R}:=\left(\beta_{n}^{\mu}\right)^{\dagger}\left(\beta_{n}^{\mu}\right)=\left(\beta_{-n}^{\mu}\right)\left(\beta_{n}^{\mu}\right)$. Further, one finds that there exist some spurious states in the theory having zero-norm and negative-norm, which are unphysical and need to be eliminated. This could be achieved using the Virasoro operators and the Virasoro algebra obeyed by them. For this we first need to understand the Fourier modes of $T_{++}$and $T_{--}$ which are as follows:

$$
\begin{aligned}
& 0=T_{++}=\left(\partial_{+} X^{\mu}\right)^{2}=l_{s}^{2} \sum_{m} L_{m} e^{-i m \sigma^{+}}, \quad L_{m}:=\sum_{n} \alpha_{m-n} \cdot \alpha_{n} \\
& 0=T_{--}=\left(\partial_{-} X^{\mu}\right)^{2}=l_{s}^{2} \sum_{m} \bar{L}_{m} e^{-i m \sigma^{-}}, \bar{L}_{m}:=\sum_{n} \beta_{m-n} \cdot \beta_{n} .
\end{aligned}
$$

where $n$ is an integer which takes all values from $-\infty$ to $+\infty$ including zero. Also, $L_{m}$ and $\bar{L}_{m}$ here are the Fourier modes of the constraints: $T_{++}=0$ and $T_{--}=0$. These modes are called as the Virasor operators and they satisfy the Virasoro algebra (with central extension):

$$
\begin{aligned}
& {\left[L_{m}, L_{n}\right]=(m-n) L_{m+n}+\frac{D}{12}\left(m^{3}-m\right) \delta_{m+n, 0}} \\
& {\left[\bar{L}_{m}, \bar{L}_{n}\right]=(m-n) \bar{L}_{m+n}+\frac{D}{12}\left(m^{3}-m\right) \delta_{m+n, 0}}
\end{aligned}
$$

Here central charge is equal to the number of spacetime dimensions $D$.This is also the number of free scalar fields on the WS. The central extension term vanishes for particular values of $\mathrm{m}$ namely, for $m=0, \pm 1$. Thus making $L_{0}, L_{1}$ and $L_{-1}$ (which form a closed sub-algebra) as somewhat special as would be seen later. Using the Legendre transformation and the above mode expansions, classical expression for the Hamiltonian for the closed string (CS) theory and open string (OS) theory could be obtained (with the sum over the integer $n$ running from $-\infty$ to $+\infty$ including $n=0$ ) to be [3]:

$$
\frac{1}{2} H=\left(L_{0}+\bar{L}_{0}\right)=\frac{1}{2} \sum_{n}\left(\alpha_{-n} \cdot \alpha_{n}+\beta_{-n} \cdot \beta_{n}\right)(C S), \quad H=L_{0}=\frac{1}{2} \sum_{n}\left(\alpha_{-n} \cdot \alpha_{n}\right)
$$

However, for the quantum ST, all the expressions need to be normal ordered. Further, any classical solution of string theory must additionally obey the infinite number of constraints: $L_{m}=\bar{L}_{m}=0$ (for $m=0, \pm 1, \pm 2, \ldots$ ). These constraints could be used to calculate the mass of a string using the relativistic mass-shell condition: $M^{2}:=\left(-p^{\mu} p_{\mu}\right)$ where $p^{\mu}$ is the total momentum of the string. For the closed string one finds $(n=1,2, \ldots \infty)$ :

$$
\begin{gathered}
L_{0}=\left[\frac{1}{2} \alpha_{0}^{2}+\sum_{n} \alpha_{-n} \cdot \alpha_{n}\right]=\left[\frac{1}{2} \alpha_{0}^{2}+N_{L}\right], N_{L}=\sum_{n} \alpha_{-n} \cdot \alpha_{n}, \quad \alpha_{0}^{2}=\left(\alpha_{0}\right)^{\mu}\left(\alpha_{0}\right)_{\mu}=\alpha^{\prime} p_{L}^{2} \\
\bar{L}_{0}=\left[\frac{1}{2} \beta_{0}^{2}+\sum_{n} \beta_{-n} \cdot \beta_{n}\right]=\left[\frac{1}{2} \beta_{0}^{2}+N_{R}\right], \quad N_{R}=\sum_{n} \beta_{-n} \cdot \beta_{n}, \quad \beta_{0}^{2}=\left(\beta_{0}\right)^{\mu}\left(\beta_{0}\right)_{\mu}=\alpha^{\prime} p_{R}^{2}
\end{gathered}
$$

Now we use the Virasoro operators for eliminating the spurious states from the theory namely, the states with the negative-norm as well as the states with zero-norm. For eliminating the spurious states with negative-norm we impose on our theory, a condition analogous to the Gupta-Bleuler 
quantization condition in quantum electrodynamics (QED) namely, we require that the expectation value of $\left(L_{0}-a\right)$ vanishes for a physical state $|\psi\rangle$ so that:

$$
<\psi\left|\left(L_{0}-a \delta_{m, 0}\right)\right| \psi>=0, \quad\left\langle\psi\left|\left(\bar{L}_{0}-a \delta_{m, 0}\right)\right| \psi>=0, m \geq 0 .\right.
$$

Presence of the term $\left(a \delta_{m, 0}\right)$ in the above expressions implies that we need the normal ordering constant $a$ only in the case of $L_{0}$ and the above relations simply imply: $\left(L_{0}-a\right)=0$ and $\left(\bar{L}_{0}-a\right)=0 \quad$ (also for the sake of completeness, the level matching condition reads: $\left(L_{0}-\right.$ $\left.\bar{L}_{0}\right) \mid \psi>=0$ or simply $\left(L_{0}-\bar{L}_{0}\right)=0$ ). Mass-shell condition for the OS theory implies:

$$
M^{2}\left|0>=\left(\frac{1}{\alpha^{\prime}}\right)(N-a)\right| 0>.
$$

Where $M^{2}$ is the mass operator of the theory. This means that the ground state (corresponding to $N=0$ ) of the bosonic OS theory has negative mass squared (for $a=1$ to be determined later) implying that it is a Tachyonic state (which is an unphysical state). It is interesting to note that the first excited state (corresponding to $N=1$ is a mass-less state (which eventually turns out to be a mass-less vector state in 26 dimensions and is analogous to the photon in the conventional 4-dimensional QED theory. Mass-shell condition for the CS theory (with $a=1$ ) similarly implies:

$$
M^{2}\left|0>=\left(\frac{2}{\alpha^{\prime}}\right)\left(N_{L}+N_{R}-2 a\right)\right| 0>\text {. }
$$

This means that the ground state for the bosonic CS theory (corresponding to $N_{L}=N_{R}=0$ ) also has negative mass squared implying a tachyonic nature for the state (which is also an unphysical state). The first excited states (corresponding to $N_{L}=N_{R}=1$ ) are seen to be massless states in $D=26$ dimensions and they include: a graviton (symmetric tensor) state, an anti-symmetric 2-form gauge field state and a scalar dilaton state (for further details see Refs. [3, 8]).

Removal of the spurious states from the spectrum of the theory could be easily understood following Ref. [3]: A state $|\psi\rangle$ is called a spurious state if it satisfies the mass-shell condition $\left(L_{0}-a\right)|\psi\rangle=0$ and if it is orthogonal to all physical states, so that $\langle\phi \mid \psi\rangle=0$ where $\mid \phi>$ represents any physical state in the theory. We consider a state (with $n=1$ to $\infty$ ) [3]:

$$
\left|\psi>:=\sum_{n} L_{-n}\right| \chi_{n}>,\left(L_{0}-a+n\right) \mid \chi_{n}>=0
$$

Using the Virasoro algebra (VA) identity: $L_{-3}=\left[L_{-1}, L_{-2}\right]$, the above state $|\psi\rangle$ could be re-expressed in the form: $|\psi\rangle=\left(L_{-1}\left|\chi_{1}\right\rangle+L_{-2}\left|\chi_{2}\right\rangle\right)$ and in general, any spurious state could be expressed in this form (cf. Ref. [3] for further details). A spurious state $|\psi\rangle$ defined as above is orthogonal to every physical state (so that: $\langle\phi \mid \psi\rangle=0$ ). Also, if a state $|\psi\rangle$ is spurious and physical then it is orthogonal to all physical states including to itself so that: $\langle\psi \mid \psi\rangle=0$ and as a consequence of this such a state has a zero norm.

Also, for a suitably chosen normal ordering constant $a$ a class of zero-norm spurious states has the form [3]: $|\psi\rangle=L_{-1}\left|\chi_{1}\right\rangle$ with $\left(L_{0}-a+1\right)\left|\chi_{1}\right\rangle=0, L_{m}\left|\chi_{1}\right\rangle=0, m>0$, and demanding that $|\psi\rangle$ is a physical state implies: $L_{m}|\psi\rangle=\left(L_{0}-a\right)|\psi\rangle=0$, for $m=1,2,3, \ldots \ldots$ Making use of the VA identity: $L_{1} L_{-1}=\left(2 L_{0}+L_{-1} L_{1}\right)$, one finds that:

$$
L_{1}\left|\psi>=L_{1} L_{-1}\right| \chi_{1}>=\left(2 L_{0}+L_{-1} L_{1}\right)\left|\chi_{1}>=2(a-1)\right| \chi_{1}>=0 .
$$


This then implies an important result namely, $a=1$, which defines the boundary between positive-norm and negative-norm states [3].

For the determination of the spacetime dimension (following the work of Ref. [3]), we consider a zero-norm spurious state: $|\psi\rangle:=\left(L_{-2}+\gamma L_{-1}^{2}\right) \mid \zeta>$ which has zero norm for a particular value of $\gamma$. The state $|\psi\rangle$ would be spurious if the state $\mid \zeta>$ satisfies the condition: $\left(L_{0}+1\right)\left|\zeta>=L_{m}\right| \zeta>=0$ for $m=1,2, \ldots$. We now impose the condition that $|\psi\rangle$ is a physical state so that it satisfies $L_{1}|\psi\rangle=0, L_{2}|\psi\rangle=0$, and $L_{m} \mid \psi>=0, m \geq 3$. Now the condition $L_{1}|\psi\rangle=0$ could be easily seen [3] to imply $\gamma=3 / 2$. Further the evaluation of $L_{2}|\psi\rangle=0$ implies that the spacetime dimension $D=26$. This could be easily seen (by using the VA with $\gamma=3 / 2$ ) as follows [3]:

$$
L_{2}\left|\psi>=L_{2}\left(L_{-2}+\frac{3}{2} L_{-1}^{2}\right)\right| \zeta>=\left(-13+\frac{D}{2}\right)=\left(\frac{-26+D}{2}\right) \mid \zeta>=0 .
$$

This leads to $D=26$ which is equal to the number of spacetime dimensions in the ST. The bosonic ST with $a=1, D=26$ is called as the critical $S T$ and $D=26$ is called as the critical dimension of the bosonic ST. In this case the zero-norm states and negative-norm states which are unphysical, decouple and all physical states have positive norm [3] as desired.

We next consider the Raymond-Nevieu-Schwarz (RNS) superstring theory (SST) in the LFC's defined by the action [3, 4], [8]:

$$
S=\left[S_{B}+S_{F}\right]=\int d^{2} \sigma\left[\mathscr{L}_{B}+\mathscr{L}_{F}\right], \mathscr{L}_{B}:=\frac{-T}{2}\left[\partial_{\alpha} X^{\mu} \partial^{\alpha} X_{\mu}\right], \mathscr{L}_{F}:=\frac{-T}{2}\left[\bar{\psi}^{\mu} \rho^{\alpha} \partial_{\alpha} \psi_{\mu}\right]
$$

Here $\psi$ is a Majorana spinor (having real components): $\psi \equiv \psi_{A}:=\psi_{\mp}:=\left[\begin{array}{l}\psi_{-}\left(\sigma^{-}\right) \\ \psi_{+}\left(\sigma^{+}\right)\end{array}\right]$where $A$ is the spinor index (with $A=\mp$ ). Here $\psi_{-}$and $\psi_{+}$are the left and right movers respectively. The Dirac matrices $\rho^{\alpha}$ are defined as:

$$
\rho^{0}:=\left(\begin{array}{cc}
0 & -1 \\
1 & 0
\end{array}\right), \rho^{1}:=\left(\begin{array}{ll}
0 & 1 \\
1 & 0
\end{array}\right), \rho^{3}=\rho^{0} \rho^{1}, \rho^{\alpha} \partial_{\alpha}=\left(\begin{array}{cc}
0 & -2 \partial_{-} \\
2 \partial_{+} & 0
\end{array}\right)
$$

The action of the RNS superstring theory in the LFC's reads:

$$
S=\int d^{2} \sigma\left[\left(T \partial_{+} X^{\mu} \partial_{-} X_{\mu}\right)+(i T)\left[\psi_{-} \partial_{+} \psi_{-}+\psi_{+} \partial_{-} \psi_{+}\right)\right]
$$

EoM following from this action are: $\partial_{+} \partial_{-} X^{\mu}=0, \partial_{+} \psi_{-}=0, \partial_{-} \psi_{+}=0$ (where the first one is the LF Klein Gordon equation and the last two are the LF Dirac equations). This theory is seen to be invariant under the global supersymmetry transformations (invented by Jervais and Sakita):

$$
\delta X^{\mu}=\bar{\varepsilon} \psi^{\mu}, \delta \psi^{\mu}=\left(\rho^{\alpha} \partial_{\alpha} X^{\mu} \varepsilon\right), \delta \bar{\psi}^{\mu}=\left(-\bar{\varepsilon} \rho^{\alpha} \partial_{\alpha} X^{\mu}\right)
$$

This invariance leads to the super conserved current for the theory to be:

$$
J_{+}=\left(\psi_{+}^{\mu} \partial_{+} X_{\mu}\right), \quad J_{-}=\left(\psi_{-}^{\mu} \partial_{-} X_{\mu}\right), \quad 0=\partial_{\alpha} J_{A}^{\alpha}=\partial_{-} J_{+}+\partial_{+} J_{-}
$$

Also, the non-vanishing components of the conserved energy-momentum tensor $0=T_{\alpha \beta}$ which results from the invariance of the SST under the translational symmetry with respect to the WS coordinates $\sigma^{\alpha}$ in LFC's are seen to be [with $0=\partial^{\alpha} T_{\alpha \beta}=\left(\partial^{+} T_{++}+\partial^{-} T_{--}\right)$]:

$$
T_{++}=\left[\partial_{+} X^{\mu} \partial_{+} X_{\mu}+(i / 2)\left(\psi_{+}\right)^{\mu} \partial_{+}\left(\psi_{+}\right)_{\mu}\right], T_{--}=\left[\partial_{-} X^{\mu} \partial_{-} X_{\mu}+(i / 2)\left(\psi_{-}\right)^{\mu} \partial_{-}\left(\psi_{-}\right)_{\mu}\right]
$$


While deriving the EoM for this SST using the variational principle, one also gets some boundary terms (BT's) which could be made to vanish by choosing appropriate BC's. There are two sets of possibilities known as: (i) Raymond (R) and (ii) Nevieu-Schwarz (NS) BC's. In the R-sector, we could e.g. choose the BC's:

$$
\psi_{+}^{\mu}(\sigma=0, \tau)=+\psi_{-}^{\mu}(\sigma=0, \tau), \psi_{+}^{\mu}(\sigma=\pi, \tau)=+\psi_{-}^{\mu}(\sigma=\pi, \tau) .
$$

whereas in the NS sector, we could choose the BC's:

$$
\psi_{+}^{\mu}(\sigma=0, \tau)=+\psi_{-}^{\mu}(\sigma=0, \tau), \psi_{+}^{\mu}(\sigma=\pi, \tau)=-\psi_{-}^{\mu}(\sigma=\pi, \tau) .
$$

These BC's are perfectly acceptable because they make the BT's disappear as desired. One has to choose the Fourier modes also in consistency with the above BC's in both the sectors. These are very well studied cases and for further details, we refer to the work of Refs.[3, 4] and Ref. [8].

It is interesting to find that the consistency in the R-sector, requires that the normal ordering constant is : $a=a_{R}=0$ with the spacetime dimensions $D=10$ and the consistency in the NS-sector, requires that the normal ordering constant is : $a=a_{N S}=1 / 2$ with the spacetime dimensions $D=10$. In R-sector, spectrum of the theory has spacetime fermions (which e.g., are described by the generalized Dirac equation in 10-dimensions) whereas in NS-sector, the spectrum has spacetime bosons. Superstring theory (in contrast with the bosonic ST) is thus seen to have the spacetime bosons as well as spacetime fermions in its spectrum which is consistent with the standard model. We refer to the work of Refs. [3, 4] and to the Video Lectures of Ref. [8] for many more details of the string theory and superstring theory.

We thank Jutta Kunz for her consistent invaluable academic support and encouragements and we thank the organizers of LC-2019 Conference (Ecole Polytechnique Palaiseau, France, Sept. 1620, 2019), Cedric Lorce and Arkadius Trawanski for their great hospitality during the conference.

\section{References}

[1] P. A. M. Dirac, Rev. Mod. Phys. 21, 392 (1949) (for Dirac's Relativistic Forms of Dynamics).

[2] S. J. Brodsky, H. C. Pauli and S. S. Pinsky, Phys. Rep. 301, 299 (1998).

[3] Katrin Becker, Melanie Becker and John Schwarz, "String Theory and M-Theory", Cambridge University Press, 2007.

[4] Kevin Wray, "An Introduction to String Theory“, Lecture Notes, May 6, 2011.

[5] Usha kulshreshtha and D. S. Kulshreshtha, Phys. Lett. B 555, 255-263 (2003).

[6] D. S. Kulshreshtha, "String Gauge Symmetries in the Light-Front Polyakov D1 Brane Action", Invited Talk at the International Conference on Light-Cone Physics (LC-2010), June 14-18, 2010, Valencia, Spain, Published in PoS LC2010 (2010) 006, 8pp.

[7] Daya Shankar Kulshreshtha, "Polyakov D1 Brane Action on the Light-Front", Invited Talk at the International Light-Cone Conference, LC-2008, held at Mulhouse, France, July 07-11, 2008, Published in PoS LC2008 (2008) 007, 6pp. (hep-th/08091398).

[8] Daya Shankar Kulshreshtha, Video Lectures on "Introduction to String Theory", University of Oldenburg, Germany, Summer Semester-2018; Daya Shankar Kulshreshtha, Video Lectures on "Introduction to Superstring Theory", University of Oldenburg, Germany, Summer Semester-2019: URL: https : //www.youtube.com/channel/UCO $O_{R} O k 6 q 8 w A I I M M A Q 7 k i_{v} A$. 